\title{
3 Research Square \\ Gut Microbiota as Prognosis Markers for Patients with HBV-Related Acute-on-Chronic Liver Failure
}

\section{Ke Wang}

Third Affiliated Hospital of Sun Yat-Sen University

\section{Zhao Zhang}

Guangdong Longsee Biomedical Corporation

\section{Zhi-Shuo Mo}

Third Affiliated Hospital of Sun Yat-Sen University

\section{Xiao-Hua Yang}

Third Affiliated Hospital of Sun Yat-Sen University

\section{Bing-Liang Lin}

Third Affiliated Hospital of Sun Yat-Sen University

\section{Liang Pen}

Third Affiliated Hospital of Sun Yat-Sen University

\section{Yang Xu}

Guangdong Longsee Biomedical Corporation

\section{Chun-Yan Lei}

Guangdong Longsee Biomedical Corporation

\section{Xiao-Dong Zhuang}

Nuffield Department of Medicine, University of Oxford

\section{Ling LV}

The First Affiliated Hospital of Nanjing Medical University

\section{Rui-Fu Yang}

State Key Laboratory of Pathogen and Biosecurity, Beijing Institute of Microbiology and Epidemiology

\section{Tao Chen}

Guangdong Longsee Biomedical Corporation

\section{zhi-liang gao ( $\nabla$ gaozhl@mail.sysu.edu.cn )}

Third Affiliated Hospital of Sun Yat-Sen University https://orcid.org/0000-0001-7611-4416

\section{Research}

Keywords: Hepatitis B, acute-on-chronic liver failure, gut microbiota, 16S rRNA sequencing, metagenomic sequencing.

Posted Date: June 9th, 2020 
DOI: https://doi.org/10.21203/rs.3.rs-32725/v1

License: (c) (1) This work is licensed under a Creative Commons Attribution 4.0 International License. Read Full License

Version of Record: A version of this preprint was published at Gut Microbes on January 1st, 2021. See the published version at https://doi.org/10.1080/19490976.2021.1921925. 


\section{Abstract \\ Background}

The gut microbiota in the hepatitis B virus related acute-on-chronic liver failure (HBV-ACLF) is poorly defined. We aim to uncover the characteristics of the gut microbiota in HBV-ACLF and in other HBV associated pathologies.

\section{Methods}

We analyzed the gut microbiome in patients with HBV-ACLF or other HBV associated pathologies and healthy individuals by $16 \mathrm{~S}$ rRNA sequencing and metagenomic sequencing of fecal samples. 212 patients with HBV-ACLF, 252 with chronic hepatitis B (CHB), 162 with HBV-associated cirrhosis (HBV-LC) and 877 healthy individuals were recruited for the study. CHB and HBV-LC patients are grouped as HBVOther.

\section{Results}

We discovered striking differences in the microbiome diversity between the HBV-ACLF, HBV-Other and healthy groups using $16 \mathrm{~S}$ rRNA sequencing. The ratio of cocci to bacilli was significantly elevated in the HBV-ACLF group compared with healthy group. Further analysis within the HBV-ACLF group identified 52 genera showing distinct richness within the group where Enterococcus was enriched in the progression group whilst Faecalibacterium was enriched in the regression group. Metagenomic sequencing validated these findings and further uncovered an enrichment of Lactobacillus casei paracasei in progression group, while Alistipes senegalensis, Faecalibacterium prausnitzii and Parabacteroides merdae dominated the regression group. Importantly, our analysis revealed that there was a rapid increase of Enterococcus faecium during the progression of HBV-ACLF.

\section{Conclusions}

The gut microbiota displayed distinct composition at different phases of HBV-ACLF. High abundance of Enterococcus is associated with progression while that of Faecalibacterium is associated with regression of HBV-ACLF. Therefore the microbiota features hold promising potential as prognostic markers for HBVACLF.

\section{Background}

Acute-on-chronic liver failure (ACLF) is a common type of end-stage liver disease characterized by rapid deterioration of underlying chronic liver disease with organ failures and high mortality[1]. Hepatitis B Virus (HBV) is a human hepadnavirus that causes acute and chronic hepatitis and hepatocellular 
carcinoma. ACLF occurs in about $30 \%$ of HBV-related cirrhosis patients with acute decompensation[2, 3]. The short-term prognosis of HBV-associated ACLF (HBV-ACLF) is poor, with 28-day mortality ranging from $40-50 \%[2-4]$.

Gut microbiota is the collection of microorganisms that inhabit in the gastrointestinal tract[5], with an estimated number of gut microorganisms of over $10^{14}[6]$. Gut microbiota has a complicated and mutually beneficial relationship with the host[7], and plays an important role in the metabolism, nutrition, pathological processes and immune function of the host[8, 9]. Human gut microbiota composition is affected by multiple factors such as age, nutrition, ethnicity, disease, and medication intake[10-12]. Intestinal microbes can produce short-chain fatty acids to improve the energy metabolism of the colon cells[13]. Some short-chain fatty acids have anti-inflammatory effects[14]. Changes in the composition of the gut microbiota have been linked to several clinical conditions, such as obesity, nonalcoholic fatty liver disease, allergic diseases, gastrointestinal diseases, autoimmune diseases and cancers[15, 16].

Growing evidences suggest that gut microbiota plays a crucial role in the induction and the progression of liver diseases[17, 18]. Bacteria and bacterial components from the gut microflora have been associated with systemic inflammation and severe liver diseases[19,20]. Translocation of gut microbe or their microbial products can induce inflammation, liver cell apoptosis and progression of liver failure[18], chronic liver disease [21] and intestinal dysfunction in liver cirrhosis[22, 23]. Chen et al shown that changes in the microbiota composition are correlated with liver disease severity in non-viral ACLF patients[17].

In clinical practice, intestinal microecological modulators are commonly used for the treatment of HBVACLF, especially for those with abdominal pain, diarrhea, hepatic encephalopathy and suspicious abdominal infection[24-26]. However, the therapeutic efficacy varies considerably likely due to the differences in gut microbiota composition. This study aims to define the composition of the gut microbiota in HBV-ACLF patients and other HBV associated pathologies including chronic hepatitis B (CHB) and HBV-associated cirrhosis (HBV-LC) and healthy individuals to uncover their relationships to disease progression and potential as prognosis markers.

\section{Results}

\section{Distinct gut microbiota distribution and genera in HBV-ACLF}

To uncover the microbiota distribution and genera in HBV-ACLF, HBV-Other and healthy groups, fecal samples were performed 16S rRNA sequencing and Shannon indexes calculated. The diversities of microbiome were significantly different between HBV-Other, HBV-ACLF and healthy group (Fig.1A). The overall gut microbiota distribution in each group was visualized using a t-distributed stochastic neighbor embedding (t-SNE) visualization and further demonstrated distinct microbiota distribution between groups, especially between the healthy and liver disease groups (Fig.1B). 
To identify the predominant gut microbiota in HBV-ACLF, LEfSe analysis was performed. The results showed that there were a number of different genera of gut microbiota between the healthy and the liver disease groups, and a trend could be observed that the HBV-ACLF had more Enterococcus relative richness than the healthy group (Fig.1C). Clinically, cocci to bacilli ratio is a common parameter used to inform the status of gut microbiota and the choice of antibiotics, therefore are often tested for patients with ACLF or abdominal and intestinal infections[27, 28]. We found that the ratio of cocci to bacilli richness was significantly different among the three groups where HBVACLF group exhibited the highest ratio (Fig.1D), suggesting the balance of gut microbiota in these patients were severely disrupted.

\section{Establishing a microbiota classification model for the healthy, HBV-Other and the HBV-ACLF group}

A classification model for the healthy, HBV-Other and the HBV-ACLF group was established by Random Forest classifier. The classification model included 18 most important taxa of the 3 groups (Fig.2A), with an AUC value of 0.89. In addition, the decomposition visualization (Fig.2B) demonstrated that the 18 selected taxa could be well distinguished among the 3 groups, suggesting the model was validly established.

\section{Correlation between clinical/demographic variable and gut microbiota}

To investigate the correlation between each clinical/demographic variable and gut microbiota among the 3 groups, adonis analysis was performed. The analysis showed that with the exception of sex, AST, $\mathrm{HBsAg}$ and $\mathrm{HBeAb}$, all the other clinical/demographic variables were significantly associated with gut microbiota differences among the 3 groups $(P<0.05$, Table 1$)$, which were consistent with previous reports[27-30].

\section{Gut microbiota taxa difference between the progression and regression groups}

To investigated whether gut microbiota differs within the HBV-ACLF group, we sub-assigned the group into progression group (disease progression at discharge; $n=47$ ) and regression group (improved outcomes at discharge; $n=165$ ) according to the Model for End-Stage Liver Disease (MELD) score at discharge. Fifty-two genera with different community richness between the HBV-ACLF progression and regression groups were identified with the most abundant genera $(p<0.005)$ listed

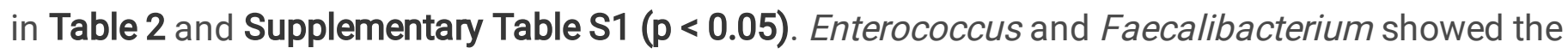
highest richness within the 52 genera, highlighting the importance of these two genera in ALCF which may contribute to disease progression. The relative abundance of Enterococcus was significantly elevated in the progression group, and that of Faecalibacterium was significantly elevated in the regression group (Fig.3).

\section{Gut microbiota genera associated with blood biochemical indicators}

To investigate whether there is a link between the gut microbiota and clinical parameters, we evaluate the association between different genera and blood biochemical indicators in all groups. The blood 
biochemical indicators were divided into three categories according to their clinical relevance as follows: Liver inflammation - alanine aminotransferase (ALT) and aspartate aminotransferase (AST); Liver disease severity - total bilirubin (TBIL), international normalized ratio (coagulation function) (INR) and end-stage liver disease model (MELD); Degree of infection - white blood cell count (WBC), neutrophil percentage (NEUT\%) and procalcitonin (PCT).

The gut microbiota genera associated with each blood biochemical indicator were identified using a Random Forest regressor via microbe's Mean Decrease Gini. We trained several

models using microbiota richness to predict their clinical relevance. By comparing the feature importance of the trained Regressor, we detected that Filifactor, Rikenellaceae, Clostridium, Bilophila and Comamonas were associated with ALT and AST(Fig.4A); Enterococcus, Enterococcaceae and Abiotrophin were associated with TBIL, INR and MELD (Fig.4B); and Enterococcus and Streptococcus were associated with WBC, NEUT\% and PCT (Fig.4C). These results have the potential to inform the use of intestinal microbial intervention to alleviate or prevent the progression of liver disease.

\section{Metagenomic sequencing between the progression and regression group in HBV-ACLF patients}

The results of genus Enterococcus and Faecalibacterium by $16 \mathrm{~S}$ rRNA sequencing were validated by the metagenomic sequencing (Fig.5A) where the richness of Enterococcus was higher in the progression group than in the regression group, and the richness of Faecalibacterium was higher in the regression group than in the progression group.

\section{The time series samples of HBV-ACLF patients}

The dynamic change of gut bacteria in patients with liver failure is an important indicator to predict the optimal time to introduce therapeutic interventions and to adjust follow-up treatments. We performed the time series samples analysis on day 1, 7 and 14 upon patient admission by metagenomic sequencing. The results showed that the richness of Lactobacillus casei paracasei was significantly higher in the progression group compared with the regression group $(P<0.05)$; while the richness of Alistipes senegalensis, Faecalibacterium prausnitzii and Parabacteroides merdae were significantly higher in the regression group $(P<0.05$, Fig.5B). The results of Faecalibacterium prausnitzii were consistent with the 16sRNA sequencing results that genus Faecalibacterium was higher in the regression group. Further analysis revealed that the regression group had a small increase in the richness of Enterococcus faecium, while the progression group had a marked increase in the richness of Enterococcus faecium during the period of 14 days. Importantly, the richness of Enterococcus was significantly higher in the progression group than the regression group in day 1 (Fig.5A).

\section{Bayes network analysis to identify the key species of gut microbiota differences}


Finally, the key species of gut microbiota which were different between the progression group and the regression group were identified using Bayes network analysis. As shown in Table 3, 7 species (Streptococcus vestibularis, Peptostreptococcus unclassified, Scardovia unclassified, Prevotella salivae, Prevotella histicola, Actinomyces odontolyticus, Streptococcus parasanguinis) were enriched in the regression group while 3 species (Ruminococcus obeum, Dorea longicatena, Clostridium citroniae) were enriched in the progression group. These results were further validated by qPCR (Fig.6). Consistently, the progression group of HBV-ACLF exhibited significantly abundant Enterococcus faecium and Lactobacillus casei paracasei, while the regression group presented significantly abundant Faecalibacterium prausnitzii, Clostridium citroniae and Dorea longicatena.

\section{Discussion}

In this study, we investigated the gut microbiota in patients with HBV-ACLF, HBV-Other (CHB, HBV-LC) and healthy individuals and our analysis demonstrated a significant difference in microbiota diversity among the HBV-ACLF, HBV-Other and healthy groups. The ratio of cocci to bacilli was significantly elevated in the HBV-ACLF group compared with the healthy group. We further identified 52 genera with different richness in the HBV-ACLF progression and regression groups. The progression group showed a high relative abundance of Enterococcus, while the regression group presented a high relative abundance Faecalibacterium. Further, metagenomic sequencing showed that the richness of Lactobacillus casei paracasei was significantly higher in the progression group than in the regression group, while Alistipes senegalensis, Faecalibacterium prausnitzii, and Parabacteroides merdae showed a significantly higher richness in the regression group than in the progression group. Further analysis revealed that Enterococcus faecium exhibited a rapid increase during the disease progression of HBV-ACLF. Taking together, these findings highlighted an important role for the composition of gut microbiota in the progression of HBV-ACLF which has important clinical implications.

Consistent with a previous report[17], our results demonstrated that gut microbiota diversity and richness were different among the HBV-ACLF group, HBV-Other group and the healthy group. Adonis analysis showed that multiple clinical/demographic variables may contribute to the differences of gut microbiota among the 3 groups, suggesting that the composition of gut microbiota was affected by multiple factors. Nevertheless, how individual factors contribute to the composition of the gut microbiota warrant further investigation.

Enterococcus is an intestinal symbiotic bacterium in healthy individuals and is emerging as an infectious drug-resistant pathogen[31]. It has been shown that the levels of Enterococcus were elevated in $\mathrm{CHB}$ and liver cirrhosis patients[32]. Our 16S rRNA sequencing showed that the ACLF progression group had a higher relative abundance of Enterococcus than the regression group, indicating Enterococcus may contribute to the progression of HBV-ACLF. Moreover, in the dynamic series of samples analysis of HBVACLFHBV-ACLFEnterococcus richness in the disease progression of HBV- ACLF. 
Faecalibacterium prausnitzii accounts for approximately $5 \%$ of total fecal microbiota in healthy adults[33]. Faecalibacterium prausnitzii depletion has been associated with several intestinal disorders including inflammatory bowel diseases[34], chronic intestinal inflammatory disorder[35], and colorectal cancer[36]. Lu et al. showed that the abundance of Faecalibacterium prausnitzii decreased in HBV-LC patients[37]. Our metagenomic sequencing showed that the abundance of Faecalibacterium prausnitzii was significantly increased in the regression group of HBV-ACLF patients, indicating that Faecalibacterium prausnitzii may be a beneficial factor of HBV-ACLF. Consistently, Enterococcus was increased in the progression group and Faecalibacterium was increased in the regression group, confirming the data generated by $16 \mathrm{~S}$ rRNA sequencing and metagenomic sequencing were highly convincing.

Clinically, the cocci to bacilli ratio of the fecal sample is often tested in HBV-ACLF patients. Most of the them showed the imbalance of cocci and bacilli, suggesting intestinal infection or gut microbiota disorder[27, 38]. Likewise, in this study, the cocci to bacilli ratio was significantly elevated in the HBVACLF group, suggesting that the HBV-ACLF patients may have cocci infection. Furthermore, this study detected an increase of Enterococcus but a decrease of Faecalibacterium in the progression HBV-ACLF group, consistent with the increased cocci to bacilli ratio in clinical findings.

Lactobacillus casei paracasei is one of the most studied and applied probiotic species of Lactobacill[39]. Nevertheless, we found that the progression group showed a higher relative abundance of Lactobacillus casei paracasei compared with the regression group, suggesting that Lactobacillus casei paracasei was associated with disease progression in HBV-ACLF and seemed contradictory to its probiotic function. Since the composition of gut microbiota is influenced by multiple factors and context dependent, the same bacterial species may play distinctive roles in different intestinal states. Therefore, the exact role of Lactobacillus casei paracasei in the progression of HBV-ACLF requires further characterization.

In the analyses of the correlation between the liver inflammationTBIL, INR, and MELD (liver disease severity); and 2 WBC, NEUT\% and PCT (degree of infection). Nevertheless, the precise causal relationships between these intestinal bacteria and biochemical parameters still require further investigation. Likewise, in our Bayes network analysis, 7 species elevated in the regression group and 3 species enriched in the progression group and the roles of these intestinal bacteria and how they contribute to disease progression remain to be investigated.

There are still some limitations to this study. First, the differences in patients' antibiotic used before and after admission may have an impact on the results of the microbiota. Secondly, the immune function and severity of liver failure at the time of admission were not consistent among the groups. Thirdly, considering the gastrointestinal symptoms and possible hepatic encephalopathy of the patients, the diet during the hospitalization was mainly based on digestible low-protein and low-fat carbohydrates, which may affect the results of microbiota. Therefore, a well-designed prospective study should be conducted to validate our findings. 


\section{Conclusions}

Our study demonstrated that the composition of gut microbiota changed at different phases of HBVACLF. High abundance of Enterococcus is associated with progression while high abundance of Faecalibacterium is associated with regression of HBV-ACLF, which is consistent with the high ratio of cocci to bacilli in HBV-ACLF patients and clinical imaging findings. The gut microbiome in HBV-ACLF patients may provide a useful prognosis marker for disease progression. Further studies should be conducted to characterize the exact roles of these gut microbiota in the progression of HBV-ACLF.

\section{Methods}

\section{Study subject}

1503 participants admitted to the Third Affiliated Hospital of Sun Yat-sen University were recruited for this study between October 2017 and November 2018 including patients with CHB $(n=252), \operatorname{HBV}-\mathrm{LC}(n=162)$ and HBV-ACLF $(n=212)$ and healthy individuals $(n=877$, from the physical examination center of the hospital). To characterize the gut microbiota, CHB and HBV-LC patients were combined and defined as the HBV-Other group. Comparative analysis was conducted among the HBV-ACLF (progression + regression) group, HBV-Other group and the healthy group. This study was approved by the institutional review board of our hospital. Written informed consent was obtained from the participants. The Medical Ethics Committee, Third Affiliated Hospital of Sun Yat-sen University (ID[2018]02-018-01).

All enrolled patients were hospitalized with HBsAg positive for $>6$ months. For CHB patients, the inclusion criteria were: alanine transaminase (ALT) $\geq 5$ upper limit of the normal (ULN), total bilirubin (TBIL) $\geq 2$ ULN, international normalized ratio (INR) $<1.5$, imaging findings (abdominal ultrasound, CT or abdominal MRI) did not support cirrhotic change. For HBV-LC patients, the inclusion criteria were: ALT $\geq 2$ ULN, TBIL $\geq 2$ ULN, INR < 1.5, imaging findings supported cirrhotic changes. HBV-ACLF was diagnosed according to the 2014 APASL diagnostic guidelines (TBIL > 5 ULN, INR > 1.5, with ascites or hepatic encephalopathy symptoms within 2 weeks).

Participants' demographic and clinical characteristics were summarized in Table 4. Age, white blood cell count, neutrophil percentage, aspartate aminotransferase, alanine aminotransferase, total bilirubin, international normalized ratio (coagulation function), procalcitonin and end-stage liver disease model were significantly different among groups.

For ethical reasons, we did not distinguish patients whether they had received antibiotics, anti-hepatitis $B$ virus and other treatments before admission. The attending doctor was free to conduct relevant medical treatment based on clinical diagnosis post-admission. HBV-ACLF patients have been routinely supplied with low-protein, low-fat diets and easily digestible carbohydrates. The use of antibiotics was only provided with symptoms including fever, abdominal pain, diarrhea, imaging based biliary infections as well as the level and ratio of white blood cells and neutrophils and procalcitonin (PCT). 


\section{S rRNA sequencing}

To analyze the gut microbiota, fecal samples of the participants were collected for 16S rRNA sequencing. The genomic bacterial DNA was extracted using Fecal Microbial Genomic DNA Extraction Kit (LS-R-N-015, Longsee biomedical corporation, China). The forward primer: 338F (5'-ACTCCTACGGGAGGCAGCA-3') and reverse primer: 806R (5'-GGACTACHVGGGTWTCTAAT-3') and sample-specific barcode sequence were used to amplify the V3-V4 highly variable region of the 16S rRNA gene (around $480 \mathrm{bp}$ ). The 16S rRNA was PCR-amplified by using Q $5^{\circledR}$ High-Fidelity DNA Polymerase (M0491, NEB, USA) according to manufacturer's protocol. Sequencing was performed by MiSeq Reagent Kit V3 (MS-102-3003, Illumina Inc., USA) using a MiSeq-PE250 sequencer (Illumina).

Bioinformatic analysis of the bacterial 16S rRNA amplicon data was conducted using a custom QIIME2 software pipeline (https://qiime2.org). Sequence quality control and filtering were conducted by FastQC v.0.11.2 and Trimmomatic v.0.32, followed by feature table construction by dada2 (Qiime2). The taxonomy of each 16S rRNA gene sequence was assigned by q2-feature-classifier (Qiime2). Pre-trained Naive Bayes taxonomy classifier gg-13-8-99-515-806-nb-classifier was used in the classification.

\section{Metagenomic sequencing}

Genomic bacterial DNA was extracted using Fecal Microbial Genomic DNA Extraction Kit (LS-R-N-015, Longsee biomedical corporation, China). PCR-amplification was performed using KAPA Hyper Plus Kit (KK8510, Kapa Biosystems, USA) and KAPA Dual-Indexed Adapter Kit (KK8722, Kapa Biosystems) followed by sequencing using NextSeq 500/550 High Output Kit v2.5 (Illumina). All procedures were performed according to the manufacturer's protocol.

For Tagenomic Sequencing Bioinformatics Analysis, sequence quality control and filtering were conducted by fastp v.0.20.0. Human genome (hg38) sequence was filtered by bowtie2. Taxonomy analysis was performed by using MetaPhIAn2 (http://huttenhower.sph.harvard.edu/metaphlan2). To identify specific species contributing to the differential genera between groups, we included 8 patients with complete HBV-ACLF (including 5 cases of regression and 3 cases of progression) for metagenomic sequencing. Fecal samples were collected at day 1 (Day-1), day 7 (Day-7) and day 14 (Day-14) after admission.

\section{qPCR validation}

Quantitative real-time PCR was used to quantify the species to validate the sequencing results. Primers were presented in Table S2. The qPCR was performed according to the PrimeScript ${ }^{\mathrm{TM}}$ RT Reagent Kit (TAKAA). Reactions were performed on a LightCycler ${ }^{\circledR}$ System (Roche, Germany) as follows: $95^{\circ} \mathrm{C}$ for 3 min, followed by 40 cycles of $95^{\circ} \mathrm{C}$ for $5 \mathrm{~s}$ and $60^{\circ} \mathrm{C}$ for $15 \mathrm{~s}$. The relative mRNA levels of target samples to control samples were calculated according to $2^{-\Delta \Delta C t}$ method, in which the difference in $\mathrm{Ct}$ values $(\Delta \mathrm{Ct})$ between the target gene and the reference gene ( $\beta$-actin) was calculated for normalization and the $\Delta \mathrm{Ct}$ of 
the different samples was compared directly $(\Delta \Delta \mathrm{Ct})$. And data were expressed as least square means \pm standard error of the mean (S.E.M.).

\section{Statistical analysis}

Mann-Whitney U test, Kruskal-Wallis test by ranks and LEfSe (Linear discriminant analysis Effect Size) analysis were conducted to identify different genus between groups. A Random Forest regressor was used to figure the genus related to certain clinical indicators by regression model's feature importance. Meanwhile, a classification model was adopted to identify a small genus set with good discriminatory power. A classification model for the healthy, CHB, HBV-LC and HBV-ACLF groups was established by using the Random Forest classifier according to the relative abundance of each genus of gut microbiota. In the model tuning process, a grid search was adopted for hyperparameter tuning, and the best score was used. Bayes network analysis was performed to figure out interaction between each species and the source of turbulence of the microbe community. Adonis (Multivariate Analysis Of Variance Using Distance Matrices) was conducted to figure the correlation between the clinical indicators and the richness of the gut microbe. A P value $<0.05$ was considered significantly different between groups. In the figures * denotes $p<0.05,{ }^{*}$ denotes $p<0.01,{ }^{* \star *}$ denotes $p<0.001$, n.s. denotes non-significant.

\section{Abbreviations}

ACLF: Acute-on-chronic liver failure; HBV: Hepatitis B Virus; HBV-ACLF: HBV-associated ACLF; CHB: chronic hepatitis B; HBV-LC: HBV-associated cirrhosis; ALT: alanine transaminase; ULN: upper limit of the normal; TBIL: total bilirubin; INR: international normalized ratio; PCT: procalcitonin; MELD: the Model for End-Stage Liver Disease; AST: aspartate aminotransferase; WBC: white blood cell count; NEUT\%: neutrophil percentage.

\section{Declarations}

\section{Acknowledgements and funding}

This work was supported by National Science and Technology Major Project (No. 2018ZX10302204), the National Natural Science Foundation of China (No. 81672701 and No. 81700528), Guangdong Basic and Applied Basic Research Foundation (No. 2019A1515110338), the Youth Innovation Project of Guangdong Province (No. 2018KQNCX101), the Science and Technology Program of Guangzhou (No. 202010060012).

\section{Authors $\square$ contributions}

Ke Wang, Zhao Zhang, Tao Chen and Zhi-Liang Gao designed the experiments, Ke Wang, Zhao Zhang, Zhi-Shuo Mo and Xiao-Hua Yang performed them. Ke Wang, Zhao Zhang, Zhi-Shuo Mo, Yang Xu, Ling Lv, Chun-Yan Lei and Xiao-Hua Yang collected and analyzed the data. The drafting of the manuscript was write by Ke Wang and Zhao Zhang, which was further Modification by Rui-Fu Yang and Zhi-Liang Gao. 
The clinical sample and information were obtained by Zhi-Shuo Mo, Xiao-Hua Yang, Bing-Liang Lin, Liang Peng. The technical, material and financial support were provided by Ke Wang, Zhao Zhang, Tao Chen, Xiao-Dong Zhuang and Zhi-Liang Gao.

\section{Ethics approval and consent to participate}

This study was approved by the Medical Ethics Committee of the third Affiliated Hospital of Sun Yat-sen University (ID[2018]02-018-01). All participants were provided a written informed consent upon enrolment.

\section{Consent for publication}

Not applicable.

\section{Competing interests}

The authors declare no competing financial interests.

\section{Author details}

${ }^{1}$ Department of Infectious Diseases, Third Affiliated Hospital of Sun Yat-sen University, Guangzhou, Guangdong 510630, China. ${ }^{2}$ Research and Development Department, Guangdong Longsee Biomedical Corporation, Guangzhou, Guangdong, China. ${ }^{3}$ Nuffield Department of Medicine, University of Oxford, Oxford OX3 7FZ, UK. ${ }^{4}$ Hepatobiliary Center, The First Affiliated Hospital of Nanjing Medical University, Nanjing, Jiangsu, China. ${ }^{5}$ State Key Laboratory of Pathogen and Biosecurity, Beijing Institute of Microbiology and Epidemiology, Beijing, China.

\section{References}

1. Zhao RH, Shi Y, Zhao H, Wu W, Sheng JF. Acute-on-chronic liver failure in chronic hepatitis B: an update. Expert Rev Gastroenterol Hepatol. 2018;12(4):341-50.

2. Li H, Chen LY, Zhang NN, Li ST, Zeng B, Pavesi M, Amoros A, Mookerjee RP, Xia Q, Xue F, Ma X, Hua J, Sheng L, Qiu DK, Xie Q, Foster GR, Dusheiko G, Moreau R, Gines P, Arroyo V, Jalan R. Characteristics, Diagnosis and Prognosis of Acute-on-Chronic Liver Failure in Cirrhosis Associated to Hepatitis B. Sci Rep. 2016;6:25487.

3. Shi Y, Yang Y, Hu Y, Wu W, Yang Q, Zheng M, Zhang S, Xu Z, Wu Y, Yan H, Chen Z. Acute-on-chronic liver failure precipitated by hepatic injury is distinct from that precipitated by extrahepatic insults. Hepatology. 2015;62(1):232-42.

4. Zheng MH, Shi KQ, Fan YC, Li H, Ye C, Chen QQ, Chen YP. A model to determine 3-month mortality risk in patients with acute-on-chronic hepatitis B liver failure. Clin Gastroenterol Hepatol. 2011;9(4):351-6 e3.

5. Thursby E, Juge N. Introduction to the human gut microbiota. Biochem J. 2017;474(11):1823-36. 
6. Backhed F, Ley RE, Sonnenburg JL, Peterson DA, Gordon JI. Host-bacterial mutualism in the human intestine. Science. 2005;307(5717):1915-20.

7. Hold GL, Hansen R. Impact of the Gastrointestinal Microbiome in Health and Disease: Co-evolution with the Host Immune System. Curr Top Microbiol Immunol. 2019;421:303-18.

8. Boullier S, Tanguy M, Kadaoui KA, Caubet C, Sansonetti P, Corthesy B, Phalipon A. Secretory IgAmediated neutralization of Shigella flexneri prevents intestinal tissue destruction by down-regulating inflammatory circuits. Journal of immunology (Baltimore, Md: 1950). 2009;183(9):5879-85.

9. Guarner F, Malagelada J-R. Gut flora in health and disease. The Lancet. 2003;361(9356):512-9.

10. Biagi E, Rampelli S, Turroni S, Quercia S, Candela M, Brigidi P. The gut microbiota of centenarians: Signatures of longevity in the gut microbiota profile. Mech Ageing Dev. 2017;165(Pt B):180-4.

11. Chen L, Zhang YH, Huang T, Cai YD. Gene expression profiling gut microbiota in different races of humans. Sci Rep. 2016;6:23075.

12. Odamaki T, Kato K, Sugahara H, Hashikura N, Takahashi S, Xiao JZ, Abe F, Osawa R. Age-related changes in gut microbiota composition from newborn to centenarian: a cross-sectional study. BMC Microbiol. 2016;16:90.

13. Flint HJ, Duncan SH, Scott KP, Louis P. Interactions and competition within the microbial community of the human colon: links between diet and health. Environ Microbiol. 2007;9(5):1101-11.

14. Macfarlane GT, Steed H, Macfarlane S. Bacterial metabolism and health-related effects of galactooligosaccharides and other prebiotics. J Appl Microbiol. 2008;104(2):305-44.

15. Clemente JC, Ursell LK, Parfrey LW, Knight R. The impact of the gut microbiota on human health: an integrative view. Cell. 2012;148(6):1258-70.

16. Sekirov I, Russell SL, Antunes LC, Finlay BB. Gut microbiota in health and disease. Physiological reviews. 2010;90(3):859-904.

17. Chen Y, Guo J, Qian G, Fang D, Shi D, Guo L, Li L. Gut dysbiosis in acute-on-chronic liver failure and its predictive value for mortality. J Gastroenterol Hepatol. 2015;30(9):1429-37.

18. Sandler NG, Koh C, Roque A, Eccleston JL, Siegel RB, Demino M, Kleiner DE, Deeks SG, Liang TJ, Heller T, Douek DC. Host response to translocated microbial products predicts outcomes of patients with HBV or HCV infection. Gastroenterology. 2011;141(4):1220-30, 30.e1-3.

19. Giannelli V, Di Gregorio V, lebba V, Giusto M, Schippa S, Merli M, Thalheimer U. Microbiota and the gut-liver axis: bacterial translocation, inflammation and infection in cirrhosis. World $\mathrm{J}$ Gastroenterol. 2014;20(45):16795-810.

20. Zeuzem S. Gut-liver axis. Int J Colorectal Dis. 2000;15(2):59-82.

21. Han DW. Intestinal endotoxemia as a pathogenetic mechanism in liver failure. World J Gastroenterol. 2002;8(6):961-5.

22. Palma P, Mihaljevic N, Hasenberg T, Keese M, Koeppel TA. Intestinal barrier dysfunction in developing liver cirrhosis: An in vivo analysis of bacterial translocation. Hepatol Res. 2007;37(1):6-12. 
23. Ramachandran A, Balasubramanian KA. Intestinal dysfunction in liver cirrhosis: Its role in spontaneous bacterial peritonitis. J Gastroenterol Hepatol. 2001;16(6):607-12.

24. Liu JE, Zhang Y, Zhang J, Dong PL, Chen M, Duan ZP. Probiotic yogurt effects on intestinal flora of patients with chronic liver disease. Nursing research. 2010;59(6):426-32.

25. Liu Q, Duan ZP, Ha DK, Bengmark S, Kurtovic J, Riordan SM. Synbiotic modulation of gut flora: effect on minimal hepatic encephalopathy in patients with cirrhosis. Hepatology. 2004;39(5):1441-9.

26. Shukla S, Shukla A, Mehboob S, Guha S. Meta-analysis: the effects of gut flora modulation using prebiotics, probiotics and synbiotics on minimal hepatic encephalopathy. Aliment Pharmacol Ther. 2011;33(6):662-71.

27. Mariat D, Firmesse O, Levenez F, Guimaraes V, Sokol H, Dore J, Corthier G, Furet JP. The Firmicutes/Bacteroidetes ratio of the human microbiota changes with age. BMC Microbiol. 2009;9:123.

28. Liu Q, Li F, Zhuang Y, Xu J, Wang J, Mao X, Zhang Y, Liu X. Alteration in gut microbiota associated with hepatitis B and non-hepatitis virus related hepatocellular carcinoma. Gut Pathog. 2019;11:1.

29. Ren YD, Ye ZS, Yang LZ, Jin LX, Wei WJ, Deng YY, Chen XX, Xiao CX, Yu XF, Xu HZ, Xu LZ, Tang YN, Zhou F, Wang XL, Chen MY, Chen LG, Hong MZ, Ren JL, Pan JS. Fecal microbiota transplantation induces hepatitis $B$ virus e-antigen ( $\mathrm{HBeAg}$ ) clearance in patients with positive $\mathrm{HBeAg}$ after long-term antiviral therapy. Hepatology. 2017;65(5):1765-8.

30. Yun Y, Chang Y, Kim HN, Ryu S, Kwon MJ, Cho YK, Kim HL, Cheong HS, Joo EJ. Alterations of the Gut Microbiome in Chronic Hepatitis B Virus Infection Associated with Alanine Aminotransferase Level. J Clin Med. 2019;8(2).

31. Low DE, Keller N, Barth A, Jones RN. Clinical prevalence, antimicrobial susceptibility, and geographic resistance patterns of enterococci: results from the SENTRY Antimicrobial Surveillance Program, 1997-1999. Clin Infect Dis. 2001;32(Suppl 2):133-45.

32. Mou H, Yang F, Zhou J, Bao C. Correlation of liver function with intestinal flora, vitamin deficiency and IL-17A in patients with liver cirrhosis. Experimental therapeutic medicine. 2018;16(5):4082-8.

33. Hold GL, Schwiertz A, Aminov RI, Blaut M, Flint HJ. Oligonucleotide probes that detect quantitatively significant groups of butyrate-producing bacteria in human feces. Appl Environ Microbiol. 2003;69(7):4320-4.

34. Sokol H, Pigneur B, Watterlot L, Lakhdari O, Bermudez-Humaran LG, Gratadoux JJ, Blugeon S, Bridonneau C, Furet JP, Corthier G, Grangette C, Vasquez N, Pochart P, Trugnan G, Thomas G, Blottiere HM, Dore J, Marteau P, Seksik P, Langella P. Faecalibacterium prausnitzii is an anti-inflammatory commensal bacterium identified by gut microbiota analysis of Crohn disease patients. Proc Natl Acad Sci U S A. 2008;105(43):16731-6.

35. De Palma G, Nadal I, Medina M, Donat E, Ribes-Koninckx C, Calabuig M, Sanz Y. Intestinal dysbiosis and reduced immunoglobulin-coated bacteria associated with coeliac disease in children. BMC Microbiol. 2010;10:63. 
36. Balamurugan R, Rajendiran E, George S, Samuel GV, Ramakrishna BS. Real-time polymerase chain reaction quantification of specific butyrate-producing bacteria, Desulfovibrio and Enterococcus faecalis in the feces of patients with colorectal cancer. J Gastroenterol Hepatol. 2008;23(8 Pt 1):1298-303.

37. Lu H, Wu Z, Xu W, Yang J, Chen Y, Li L. Intestinal microbiota was assessed in cirrhotic patients with hepatitis B virus infection. Intestinal microbiota of HBV cirrhotic patients. Microb Ecol. 2011;61(3):693-703.

38. Zhang Y, Zhao R, Shi D, Sun S, Ren H, Zhao H, Wu W, Jin L, Sheng J, Shi Y. Characterization of the circulating microbiome in acute-on-chronic liver failure associated with hepatitis B. Liver Int. 2019;10.1111/liv.14097.

39. Hill D, Sugrue I, Tobin C, Hill C, Stanton C, Ross RP. The Lactobacillus casei Group: History and Health Related Applications. Front Microbiol. 2018;9:2107.

\section{Figures}


A

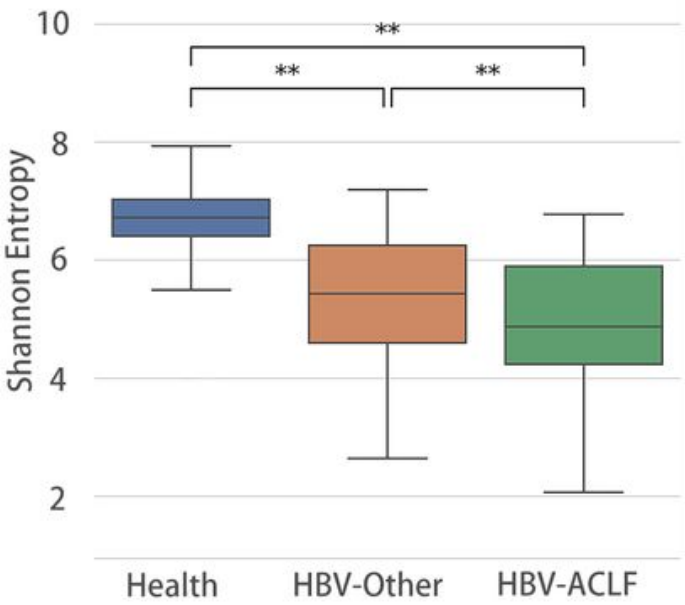

C

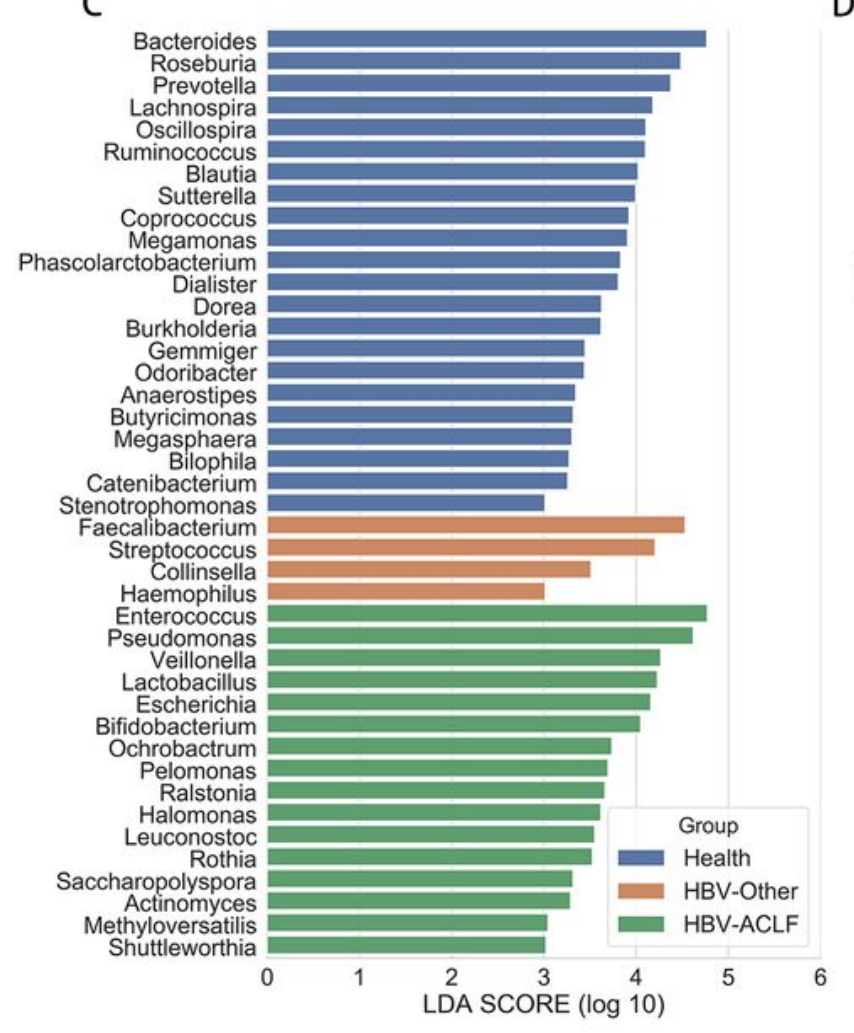

B

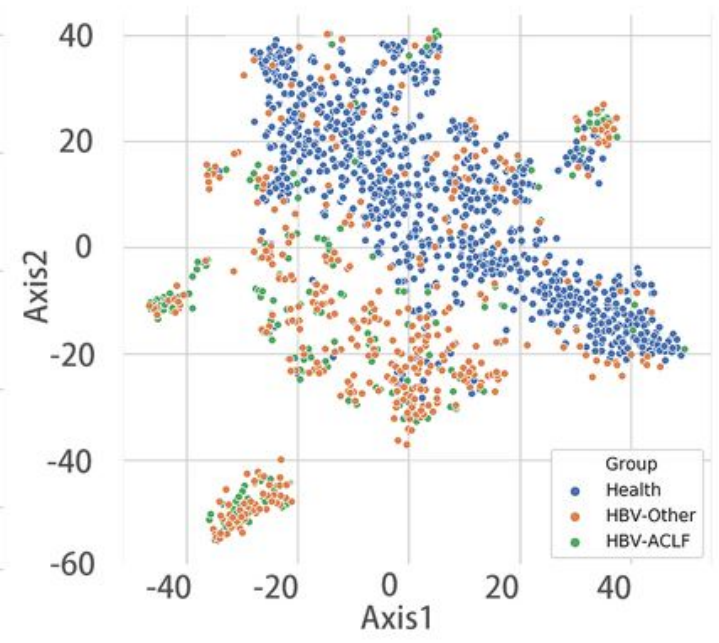

D

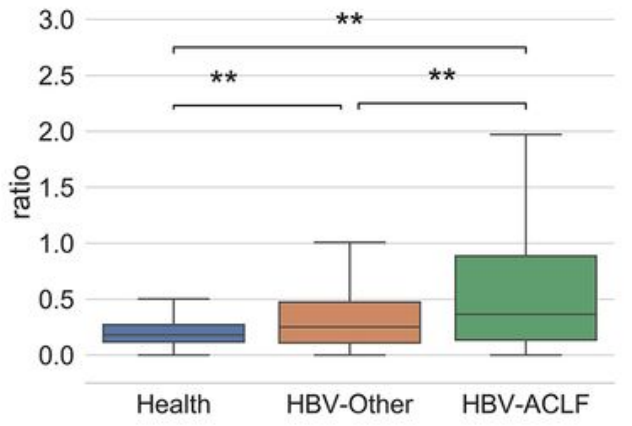

\section{Figure 1}

Gut microbiota distribution among groups. (A) Alpha diversity analysis. (B) A t-distributed stochastic neighbor embedding (t-SNE) visualization. (C) Lef Se analysis showed predominant gut microbiota. (D) The ratio of cocci to bacilli was compared among the three groups. ${ }^{\star *} \mathrm{P}<0.01$. 
A

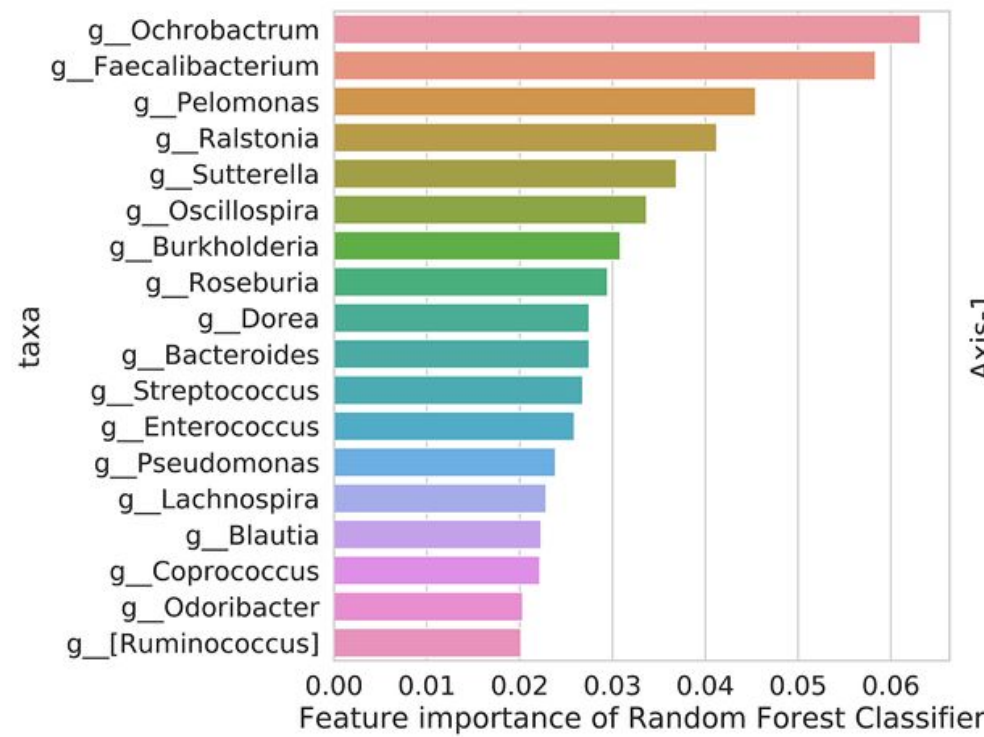

B

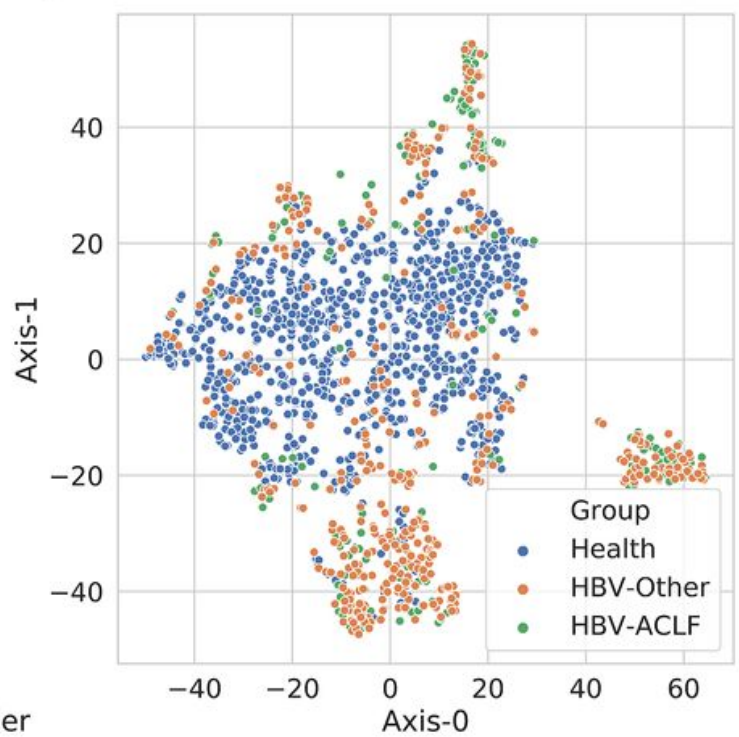

Figure 2

A classification model for the healthy, HBV-Other and the HBV-ACLF group. (A) 18 most important taxa in the classification model among the 3 groups. (B) The decomposition visualization of the 18 most important taxa among the 3 groups.

g_Enterococcus

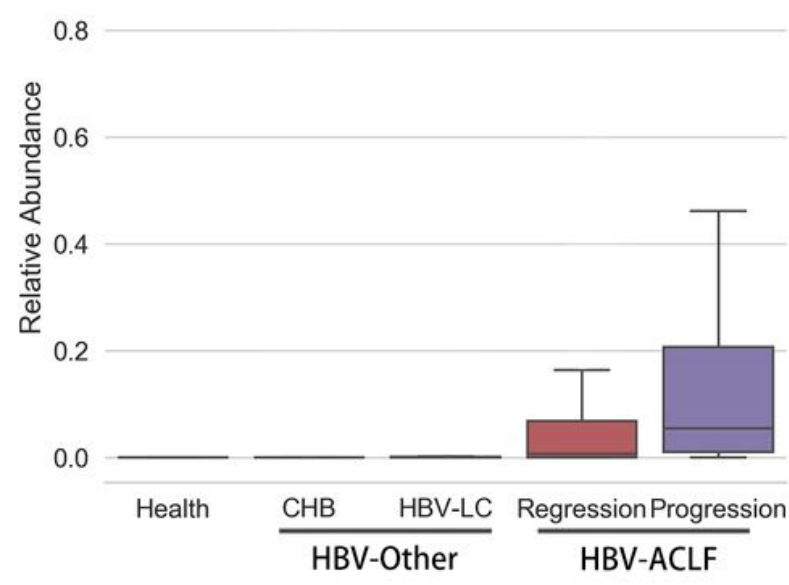

g_Faecalibacterium

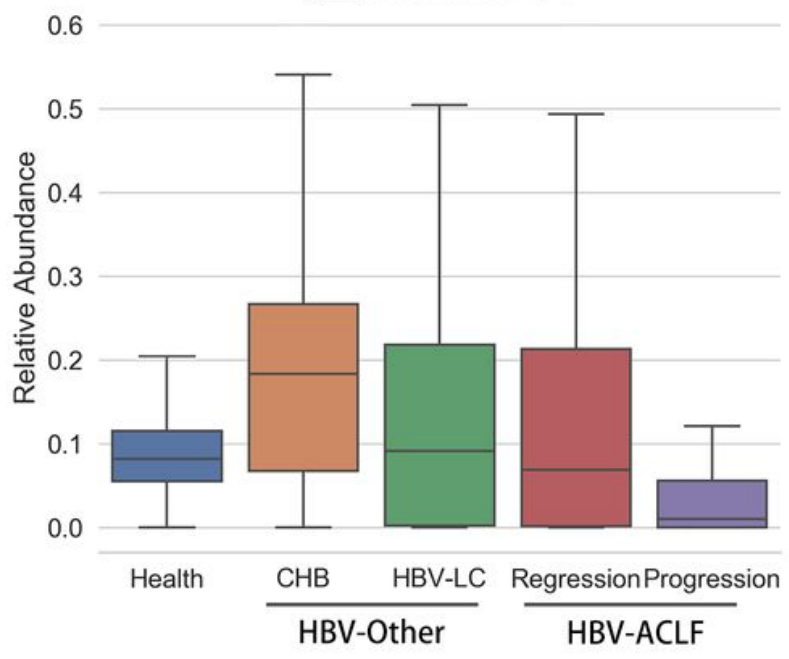

\section{Figure 3}

The abundance of the genera with the highest richness Enterococcus and Faecalibacterium. The relative abundance of Enterococcus was significantly elevated in the progression group, and that of Faecalibacterium was significantly elevated in the regression group. 


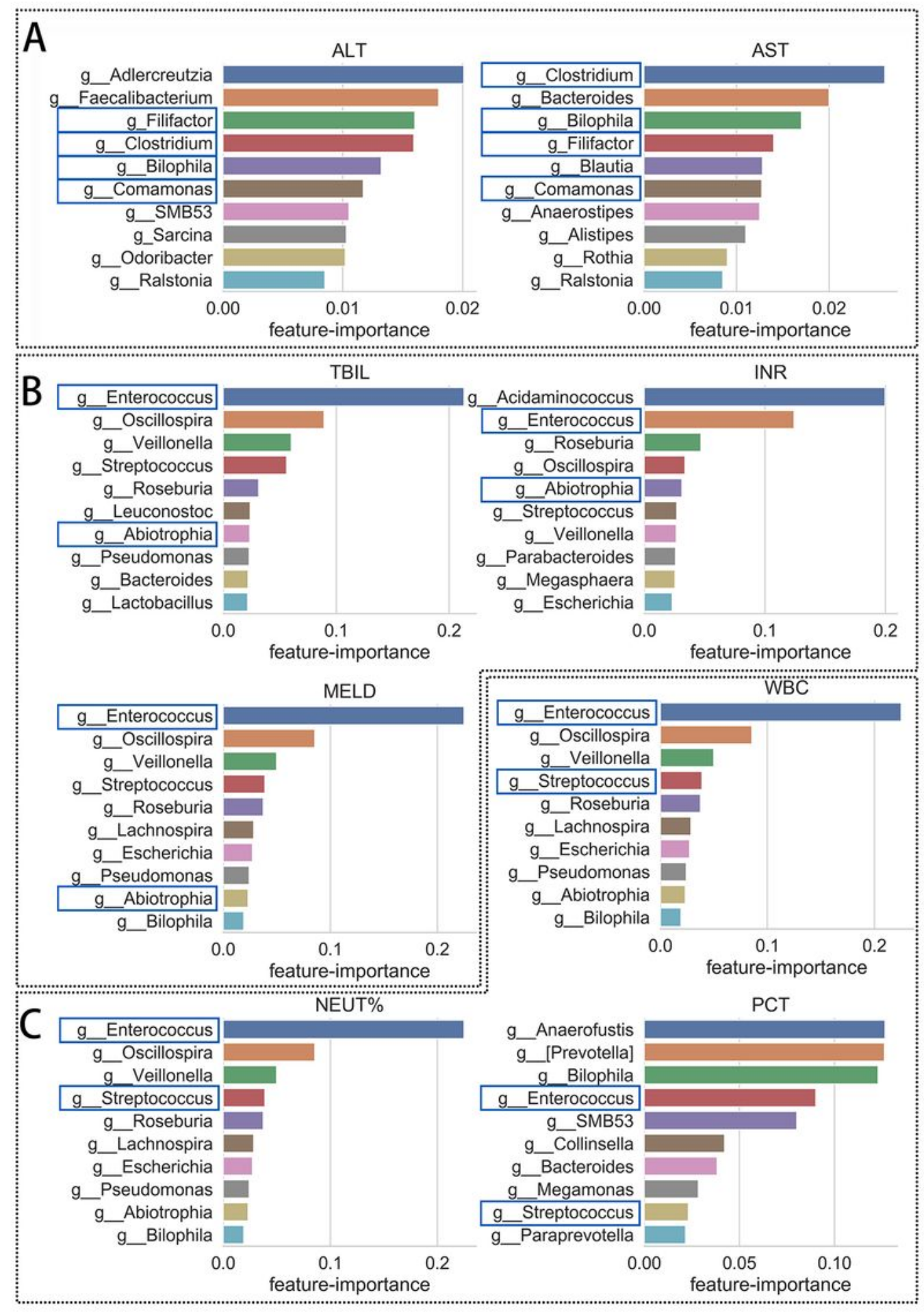

\section{Figure 4}

Correlation between the gut microbiota and clinical indicators. The genera associated with ALT/AST (relevant to liver inflammation), TBIL/INR/MELD (relevant to liver disease severity) and WBC/NEUT\%/PCT (relevant to the degree of infection). Blue square represents the genera selected by the trained Regressor that with clinical relevant. 
A

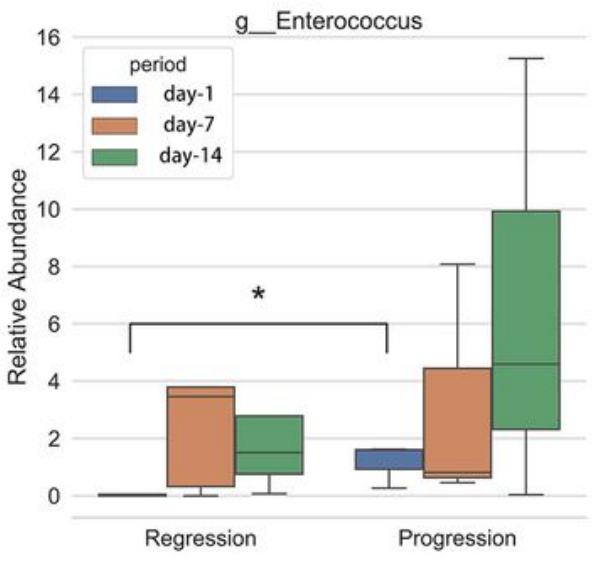

B
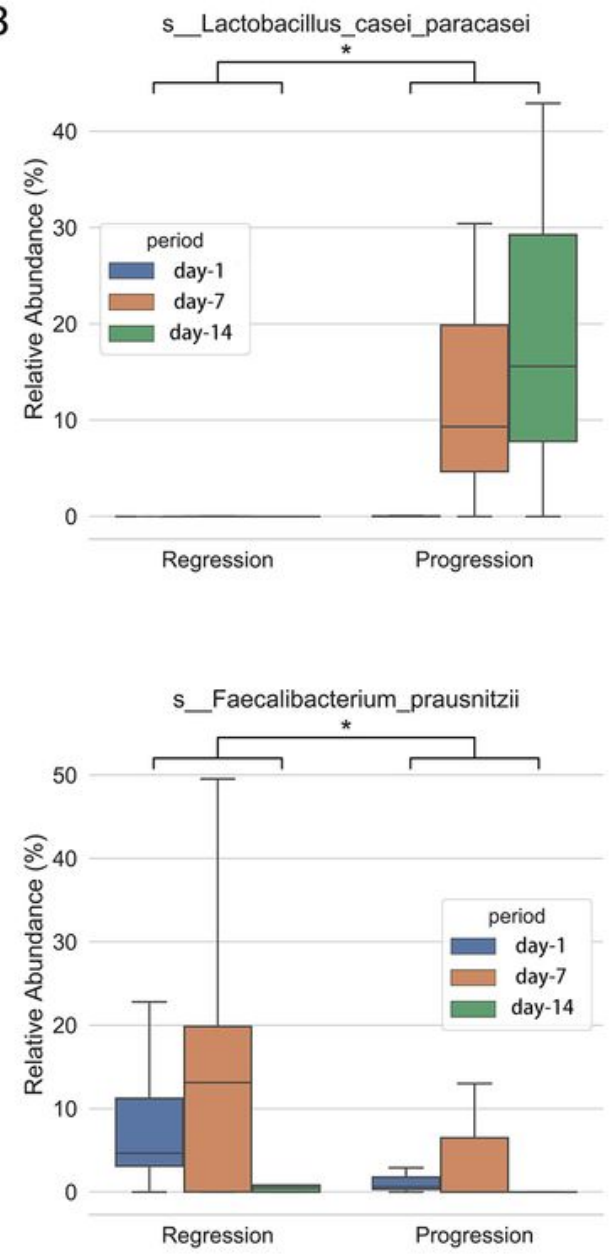
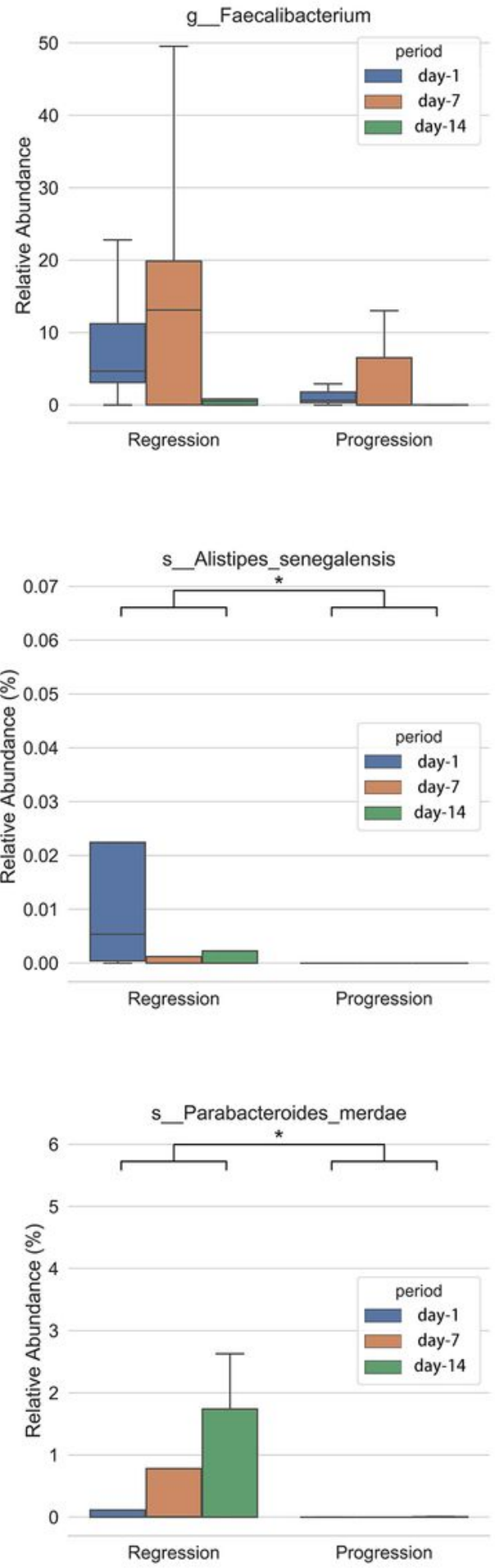

\section{Figure 5}

Difference of gut microbiota between the progression and regression groups. Relative abundance of genus at day- 1 (fecal sample collected at day 1 after admission), day 7 and day 14 between the progression and regression group of HBV-ACLF. (A) Enterococcus and Faecalibacterium, and (B) species Lactobacillus casei paracasei, Alistipes senegalensis, Faecalibacterium prausnitzii and Parabacteroides merdae. ${ }^{* *} \mathrm{P}<0.05$. 

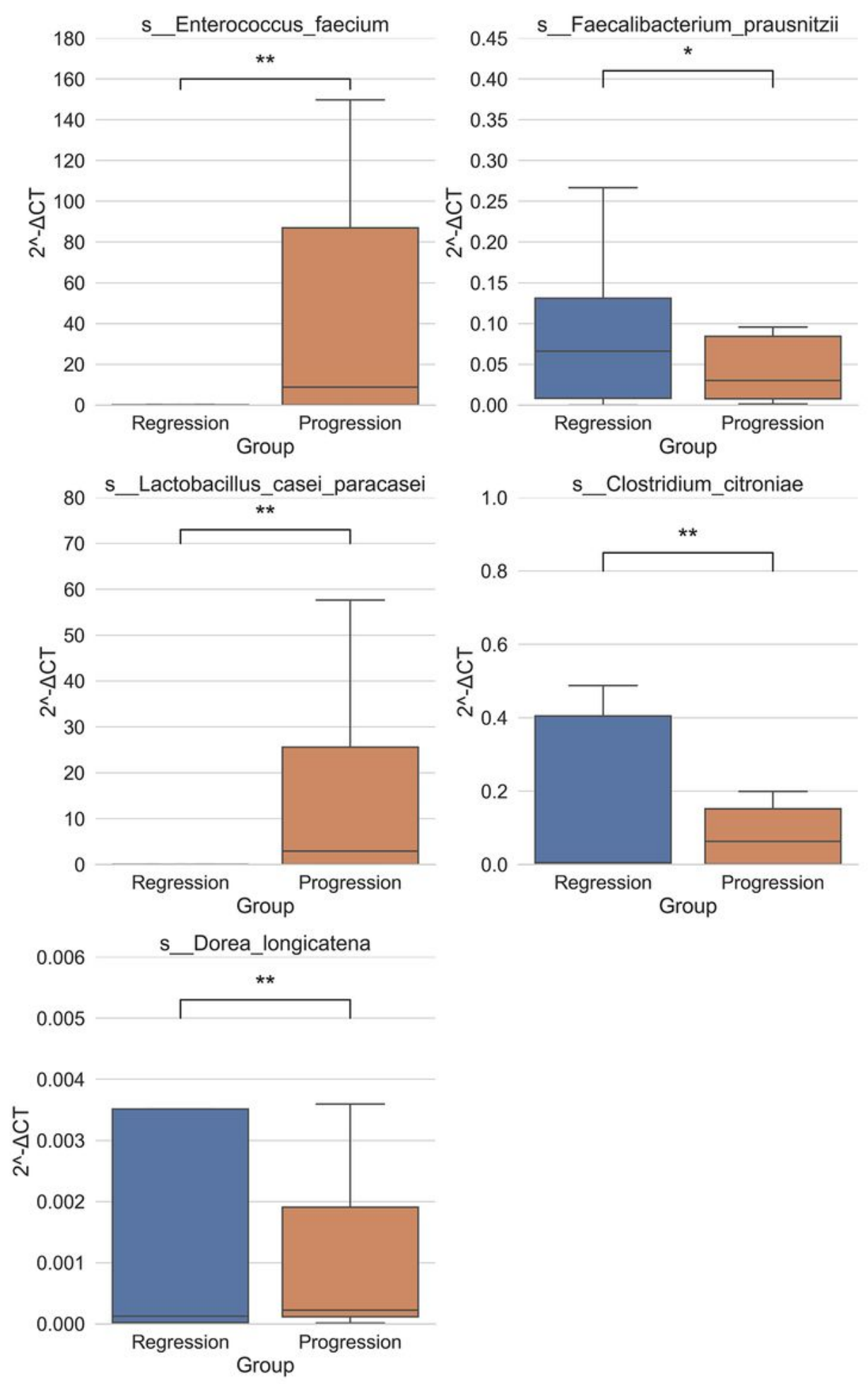

\section{Figure 6}

The key species of different gut microbiota were further validated by qPCR. qPCR validation of the relative abundance of Enterococcus faecium, Faecalibacterium prausnitzii, Lactobacillus casei paracasei, Clostridium citroniae and Dorea longicatena between progression and regression group of HBV-ACLF. $\star * P<0.01$. 


\section{Supplementary Files}

This is a list of supplementary files associated with this preprint. Click to download.

- Tables2.docx

- Tables1.docx 